\title{
Relation Between Fourier and Taylor Series
}

\author{
A. Guha ${ }^{1,2}$
}

Received: 17 October 2015/Revised: 7 July 2016/Accepted: 5 December 2016/Published online: 19 January 2017

(c) The Author(s) 2017. This article is published with open access at Springerlink.com

\begin{abstract}
Infinite series can converge in various ways to give the resultant function. Particularly, here, we consider the Fourier series and compare it with its Taylor equivalent both of which are convergent infinite series in their own rights. However, these are valid under separate limiting conditions. We consider what happens if we try to derive one series from the other or see if such a derivation is possible at all and its implications. An expansion of the Dirichlet kernel, while using a form of the Dirac delta function has been shown to yield the Taylor series in its form. However, it introduced certain restrictions on both the local and global nature of such a function.
\end{abstract}

Keywords Fourier series - Taylor series · Dirichlet kernel · Dirac delta function $\cdot$ Heaviside step function

\section{A. Guha}

ag922@cam.ac.uk

1 Department of Physics, St. Stephen's College, University of Delhi, New Delhi, India

2 Institute of Astronomy, University of Cambridge, Cambridge, UK
The foundation of Fourier series ${ }^{1}$ is based on the principle of orthogonality of functions. Now, the coefficients for the Fourier Series can easily be derived by using the orthogonality condition. If we try to apply a similar analogy to Taylor Series coefficients, then the function which obtains all the Taylor coefficients turns out to be not quite a function, but a distribution called the Dirac delta function. This will explicitly be used later to derive one from the other later.

The Fourier series can be succinctly represented in the form of the Dirichlet kernel as:

$$
\begin{aligned}
f(x) & =\lim _{r \rightarrow \infty} \frac{1}{2 \pi} \int_{-\pi}^{\pi} f(t) \Sigma_{n=-r}^{r} e^{i n(x-t)} d t \\
& =\lim _{r \rightarrow \infty} \frac{1}{2 \pi} \int_{-\pi}^{\pi} f(t) \frac{\sin (2 r+1) \frac{x-t}{2}}{2 \sin \frac{x-t}{2}}
\end{aligned}
$$

Using a certain representation of the Dirac delta function that suits us:

$$
\begin{aligned}
\delta_{n}(x) & =\frac{1}{2 \pi} \int_{-n}^{n} e^{-i x t} d t \\
& =\frac{\sin n x}{\pi x}
\end{aligned}
$$

Therefore, as a slight modification, the Dirac delta can be represented (in the limit of large $\mathrm{n}$ ) as:

$$
\delta_{n}(x)=\frac{1}{2 \pi} \frac{\sin (n+1 / 2) x}{\sin 1 / 2 x}
$$

Introducing a change of variables with $s=x-t$, we solve the above integral by repeatedly using by parts. Performing the first 2 iterations gives us:

\footnotetext{
${ }^{1}$ If the function satisfies the Dirichlet conditions.
} 


$$
\begin{aligned}
f(x)= & \lim _{r \rightarrow \infty} \frac{1}{2 \pi}\left[f(x+s) \int_{-\pi-x}^{\pi-x} \frac{\sin (r+1 / 2) s}{\sin s / 2} d s\right. \\
& \left.-\int_{-\pi-x}^{\pi-x} f^{\prime}(x+s)\left(\int \frac{\sin (r+1 / 2) s}{\sin s / 2} d s\right) d s\right]
\end{aligned}
$$

The integration of the Dirac delta function gives the Heaviside step function where it is defined as: ${ }^{2}$

$$
\theta(x) \equiv \int_{-\infty}^{x} \delta(s) d s
$$

A notation is thus introduced here so that we can sufficiently represent the successive integrals of the Heaviside function as we expand similarly the other terms, continually invoking by parts. $\theta_{0}(x)$ is the Heaviside function while $\theta_{1}(x), \theta_{2}(x), \ldots, \theta_{n}(x)$ represent the first, second, ... and nth integrals of the Heaviside function respectively.

Because of the nature of the Heaviside function, we note immediately that the function with successive integrations performed will only resemble the function in the interval $-\pi$ to $\pi$. Thus after a few iterations, we obtain:

$$
\begin{aligned}
f(x)= & f(x+s)-f^{\prime}(x+s) \int_{-\pi-x}^{\pi-x} \theta_{0}(s) d s \\
& +f^{\prime \prime}(x+s) \int_{-\pi-x}^{\pi-x} \theta_{1}(s) d s \\
& -f^{\prime \prime \prime}(x+s) \int_{-\pi-x}^{\pi-x} \theta_{2}(s) d s+\cdots
\end{aligned}
$$

Now, the integrations of these class of $\theta$ functions can be performed trivially to obtain as follows:

$$
\begin{gathered}
\theta_{1}(x)=x \theta_{0}(x) \\
\theta_{2}(x)=\frac{x^{2}}{2} \theta_{0}(x) \\
\ldots
\end{gathered}
$$

Further, the above integrals are limited between $-\pi-x$ to $\pi-x$. This thus means we only need to consider the $-(-\pi-x)$ term. In the portion to the left of limits placed, the $\theta$ functions remain uniformly zero while to the right of the limit, we obtain functions which are a difference of the $2 \theta$ functions at limit values, giving us a function one order lesser than required.

Thus on expanding, it looks very similar to the Taylor series, but not quite of the same form. However, on closer inspection, we get the same form between $-\pi$ to $\pi$.
Thus going on expanding the last term by parts over and over, we get:

$$
\begin{aligned}
f(x)= & f(x+s)-f^{\prime}(x+s)(-\pi-x)+\frac{f^{\prime \prime}(x+s)(-\pi-x)^{2}}{2 !} \\
& -\frac{f^{\prime \prime \prime}(x+s)(-\pi-x)^{3}}{3 !}+\frac{f^{\prime \prime \prime \prime}(x+s)(-\pi-x)^{4}}{4 !}-\cdots
\end{aligned}
$$

Transforming:

$s:=(-\pi-x)=-h$

We thus obtain:

$f(x)=f(x-h)+\frac{h f^{\prime}(x-h)}{1 !}+\frac{h^{2} f^{\prime \prime}(x-h)}{2 !}+\cdots$

Specifically:

$f(\pi+h)=f(\pi)+\frac{h f^{\prime}(\pi)}{1 !}+\frac{h^{2} f^{\prime \prime}(\pi)}{2 !}+\cdots$

This shows us that the value of the function and its derivatives at $\pi$ is extremely important. This can then be further generalised to a Fourier series with any suitably well-defined period. Thus the Taylor series is shown to be exactly derivable from its Fourier series. However there are a few important caveats which are listed below.

It must be noted that the above formulation of equality holds only for the specific transformation relation. Generalizing, this shows that the global properties of Fourier series can be matched by the local properties of expanding a function using a certain expansion coefficient. This above formulation could be replaced for a general Fourier series with the period replacing the limits $-\pi$ to $\pi$.

Thus, a neat relation has been shown to exist between a function's Fourier and Taylor series in a certain domain.

Open Access This article is distributed under the terms of the Creative Commons Attribution 4.0 International License ( http://creativecommons.org/licenses/by/4.0/), which permits unrestricted use, distribution, and reproduction in any medium, provided you give appropriate credit to the original author(s) and the source, provide a link to the Creative Commons license, and indicate if changes were made.

\footnotetext{
${ }^{2}$ Actually there is some subtlety regarding the definition of the Heaviside function exactly at zero. But we'll not need it for our current purposes.
} 